\title{
Food additives and cancer
}

By Frank A. Fairweather, Director, DHSS Toxicology Unit, Dominion House, 59 Bartholomew Close, West Smithfield, London $E C_{\mathrm{I}} A{ }_{7} B E$ and CHERYL A. Swann, Department of Health and Social Security, Market Towers, I Nine Elms Lane, Vauxhall, London $S W 8{ }_{5} N Q$

Food additives have been in use since prehistoric times. Early additives included salt, honey, wine and spices, all used largely for preservative or flavouring purposes. Later methods of preserving food included salting and smoke-curing of meats to enable an all round year's supply. In recent times there has been a considerable increase in the number and nature of additives available; they are used for many purposes and in the long run permit reduced food wastage, better food production and distribution throughout the world and the use of convenience foods in an urbanized community. The use of food additives is governed by certain principles, namely that they should be technologically effective, safe-in-use, not be used in any greater quantities than is necessary, not be used with the intention of misleading the consumer as to the nature or quality of the food and that the use of non-nutrient additives be kept to a minimum. The assessment of safety-in-use of food additives covers many aspects including prevention of the consumer from exposure to carcinogens.

Cancer is not a rare disease but a common one; neither is it new but has been recognized since antiquity. But cancer in the industrialized world today is found to be occurring in organs which in the past were neither previously associated with cancer nor are those in which cancer is now found to be occurring in the less welldeveloped nations. Thus, there is an indication of the association of some cancers with the changing environment and it has been suggested that this may include the increased use of food additives. However, the consumer should not ignore the fact that if the use of food additives ceased many other natural substances possessing possible carcinogenic activity would continue to be ingested.

The risk of carcinogenicity associated with recently approved food additives is very much lower than that from those in use for longer than approximately 30 years. The reason for this is that all recently approved additives must have shown adequate evidence of a lack of carcinogenicity in animal experiments whereas substances used for many years have only recently (the last decade or two) been subjected to carcinogenicity tests considered to be of adequate design. The coal tar dyes are a good example of this. Their use as food colours began in $185^{6}$; they were brighter and more concentrated than the natural colourings used until then. However, as more and more results from animal experiments indicated possible carcinogenic risks to the consumer their use gradually became phased out.

In extrapolating the findings of a carcinogenicity study the significance of $0029-6651 / 81 / 4013-4504 \$ 01.00$ (c) 1981 The Nutrition Society 
tumour formation in the experimental animal study must be assessed. Long-term testing in laboratory animals is still the best method for determining carcinogenicity although it is considered prudent nowadays to precede this with short-term in vitro screens. If at this screening stage a positive result is obtained the propriety of further long-term testing must be seriously questioned. Should the results of a long-term carcinogenicity test produce an effect in which tumour incidence is dose-related, the induction period is shortened and there is an increase in the number of tumours over control animals then the compound is of great carcinogenic risk and should not be used in foods. However, if the increase in tumour incidence is only very slight and there is no shortening of the induction period then the question must be posed: 'What is the significance of these results?' Perhaps further studies are called for, but in the meantime what precautions should be taken? This problem is one for the groups of people involved in safety evaluation. The over-all safety of food additives in the UK is the responsibility of both government and industry. The government is advised on the safety-in-use of these substances by independent expert committees who evaluate the results provided both by industry and that obtained from the published literature. Many aspects are considered namely the additive's purpose, effectiveness and information relating to toxicity and carcinogenicity. Thus an assessment may be made of the risks $v$. the benefits of each individual additive. Therefore, it is important for there to be good collaboration between government and industry to enable the final evaluation to be correct.

The finding of carcinogenicity has been the main, but not the only, toxicological effect prompting legislative decisions or further additional research or both and this paper briefly discusses some examples of food additives linked with cancer in animal studies, the testing methods used and the problems these findings have posed, bearing in mind that food additives may cause cancer by a number of different routes other than 'direct'. Some proposed examples of this are; ( $\mathrm{I}$ ) there may be an impurity present-for a long time this was believed to be the case with saccharin, (2) the additive may react with a food constituent to give a new compound, e.g. formation of nitrosamines, (3) the additive may be converted metabolically by the animal or its gut bacteria to another compound, e.g. the conversion of cyclamate to cyclohexylamine.

\section{Furyl furamide ( $A F-2)$}

AF-2 was used as an antimicrobial preservative in Japan between 1965 and 1974 in products such as fishcakes, fish and meat sausages, soybean curd and red bean paste. No carcinogenicity was detected when AF-2 was given the go-ahead for use as a food additive although tests, whose design and performance were appropriate at the time, were carried out as part of the safety evaluation programme. On the basis of mutagenicity results, published in 1972, from several test systems (increased chromosomal aberrations in cultured human lymphocytes and strong mutagenicity with $E$. coli) carcinogenicity studies in animals were initiated. Two years later carcinogenicity was demonstrated. Male $d d Y / S L C$ mice given diets 
containing $0.15-0.45 \%$ AF-2 showed a dose-related increase in squamous cell carcinomas of the forestomach.

Since carcinogenicity was first reported in animals by Ikeda and coworkers in an unpublished study in 1974 several studies have been undertaken to investigate this phenomenon. Mice and hamsters given AF-2 in the diet developed squamous cell carcinomas of the forestomach in less than I year. In mice the latent period appeared to be slightly shorter. Forestomach papillomas were identified in those rodents given doses of $\mathrm{AF-2}$ insufficient to produce squamous cell carcinomas (Sano et al. 1977; Yokoro et al. 1977; Kinebuchi et al. 1979.) AF-2 was also found to result in hepatic enlargement and consistent degenerative hepatic changes in treated dams and their offspring in a three-generation feeding study in mice (Imahori et al. I977). In contrast, the administration of AF-2 to rats resulted in formation of mammary adenocarcinomas (Cohen et al. 1977; Takayama \& Kuwabara, 1977), although Takayama \& Kuwabara did note forestomach tumours in rats killed at the end of the study (month $I 8$ ).

The important issue here is that the carcinogenic potential of AF-2 was initially detected in short-term in vitro screening tests thus emphasizing that the results of such tests should not be overlooked. This particular material also demonstrated that the same type of tumour would not necessarily be produced in a variety of experimental animal strains and emphasizes the importance of careful pathological techniques - an area sadly neglected in the past.

\section{Safrole}

Safrole is a flavouring material and is a major constituent of sassafras, star anise and camphor oils and a minor constituent of oil of nutmeg and cinnamon leaf oil. Homburger et al. ( $196 \mathrm{I}$ ) reported the induction of hepatic tumours in rats fed $\mathrm{I} \%$ safrole in the diet for $150-360 \mathrm{~d}$. Tumour induction was preceded by cirrhosis and accumulation of massive deposits of ceroid pigment. These effects were not demonstrated at a dietary level of $0.1 \%$. The findings were confirmed by Long et al. (1963) using a level of $0.5 \%$ safrole. No tumours were found in any other organ and those observed were both benign and malignant (hepatic cell adenoma, hepatic cell carcinoma, hepatocholangioma and hepatocholangiosarcoma). Hepatic cell enlargement was also seen with irregular coarse and fine nodularity and cyst formation. In the rat and mouse safrole is metabolized to $\mathbf{I}^{\prime}$-hydroxysafrole. Borchert et al. (1973) carried out both oral and subcutaneous administration studies with $I^{\prime}$-hydroxysafrole and $I^{\prime}$-acetoxysafrole in both rats and mice and showed the I'-hydroxysafrole to be a proximate carcinogen and that it was more carcinogenic than safrole itself. Investigation by Wislocki et al. (1977) of the mutagenicity of a series of compounds related to safrole revealed significant mutagenicity in Salmonella typhimurium strains TA I535 and TA 100 by $\mathbf{I}^{\prime}-$ acetoxysafrole and the $2^{\prime}-$ and $3^{\prime}$-oxides of safrole. Mutagenicity was also shown with the $2^{\prime}$-and $3^{\prime}$-oxides of $I^{\prime}$-hydroxysafrole, I'-acetoxysafrole and $I^{\prime}$-oxosafrole. The rodent metabolite $I^{\prime}$-hydroxysafrole was found not to be mutagenic.

Benedetti et al. (1977) compared the metabolism of single oral doses of up to 
$0.6 \mathrm{mg} / \mathrm{kg}{ }^{14} \mathrm{C}$-safrole in rat and man and revealed rapid absorption followed by excretion, almost entirely within $24 \mathrm{~h}$, in the urine. Increasing the dose resulted in a reduction in elimination rate and accumulation of the compound in the liver and kidney. The main urinary metabolite in both species was shown to be $\mathrm{I}, 2-$ dihydroxy-4-allylbenzene, excreted in a conjugated form. The proximate carcinogen, $I^{\prime}$-hydroxysafrole, and $3^{\prime}$-hydroxysafrole were detected as conjugates in the urine of the rat only and not in man. Bearing this latter observation in mind, the question must be posed, would safrole cause cancer in man?

Human exposure to safrole cannot be stopped completely because as a natural substance it will always be present to some degree. However, the case of safrole shows how important it is to focus attention on comparative metabolism between test animals and man. If it is proven that the known carcinogen is not excreted in man then common sense should prevail in interpretation of the results. Although comparison of the mutagenic and carcinogenic effects of the rodent metabolite $\mathrm{I}^{\prime}$ hydroxysafrole did not reveal any apparent correlation, the mutagenicity tests applied were limited; for a valid statement to be made regarding the ability of short-term in vitro screening tests to predict carcinogenicity, a greater range of in vitro screens should be applied.

\section{Colours}

Colour additives have received special attention over the years largely because their reasons for use as food additives needed greater justification than those for antioxidants and preservatives. In addition, the use of many colours has been discontinued during the last decade, based on the results of animal studies. One of the first colours to be discontinued was butter yellow, which was used in margarine until $194^{\circ}$ when it was shown to induce hepatomas in the rat. Subsequent studies showed that this effect was limited to the rat only.

The question of the adequacy of the testing and analytical procedures is often raised by toxicologists. Some years ago many colours were tested by subcutaneous injection; this route of administration was recommended in cases where compounds were poorly absorbed and thus ensured that an adequate concentration of the test substances reached the body tissues, irrespective of their metabolic fate. As a result of this procedure substances not shown to be carcinogenic by the oral route were sometimes shown to induce subcutaneous sarcomas at the injection site, especially the triphenylmethane dyes. It thus became essential to distinguish between instances in which the sarcomas developed as an artifact arising from the route of administration and those which were truly due to the carcinogenic potential of the material. Following work carried out at BIBRA (Grasso \& Golberg, 1966; Grasso et al. 1971) it became generally accepted that this route of administration was inappropriate for the safety assessment of food additives.

The banning of the colour amaranth serves as a good illustration of decisions being taken on the basis of poorly designed and conducted studies. Amaranth was banned in the USA early in 1976 when a significantly increased number of tumours in female rats only was shown following a statistical re-evaluation of previously 
accepted results. Questions about the safety of amaranth with respect to its effects on reduced fertility and lactation and its carcinogenic potential were raised early in the 1970 os by the results from studies carried out in the USSR. To try to answer these questions two major studies were then initiated in the USA. The US study on which the ban was ultimately based was of a very poor standard in two respects; (I) post-mortems of dead rats were not carried out in sufficient time to prevent serious autolysis, (2) at one stage in the study several rats were placed in the wrong cages thus involving interchange of some treatment and control animals.

The controversial statistical analysis of the study revealed that although the total number of tumours did not increase, the ratio of malignant to benign tumours did increase in females given diets containing $3 \%$ amaranth (the highest dose). The US admitted that it did not claim amaranth to be a carcinogen but that safety had not been demonstrated, although it did admit that there was no evidence of a public health hazard. Interestingly enough few countries followed the American action.

Thus, the history of the use of food colours indicates three important points which should be borne in mind when making an assessment of the safety-in-use for any material; (I) the route of administration of the test substance should be the same as that in man. Therefore the subcutaneous administration studies with colours are inappropriate for an evaluation of their safety-in-use as food additives although the results of these studies may be relevant in connection with certain medical and cosmetic uses of these substances, (2) good laboratory practice is of paramount importance in all studies and decisions on safety-in-use of food additives should not be based on poorly conducted studies, (3) statistical evaluations of tumour results can vary enormously. Depending on the way in which the analysis is selected and applied, vastly different conclusions may be drawn. Therefore, it is important where possible to standardize all statistical methods.

\section{Artificial sweeteners}

The banning of cyclamates raised significant questions about the validity of testing methods and the interpretation of results. Cyclamates, it was claimed, when fed to rats in the ratio ro:I cyclamate-saccharin, caused an increase in bladder tumours in rats. Evidence at that time had pointed to an association between cyclamates and bladder cancer; together with the fact that saccharin had been in use for many years without any evidence of hazard to human health, it was decided to ban cyclamates first in the USA in 1969 and shortly afterwards in many other countries. Saccharin, on the other hand, continued to be used.

Saccharin, synthesized in 1879 from toluene, became available commercially from I 900 onwards. Its use in soft drinks increased significantly following the Second World War when diet sodas were introduced in response to a weight conscious population. The long-term safety of saccharin has been studied in various animal experiments since 1950 . Currently at least twenty studies have been performed in rats, mice, hamsters and monkeys. Of these studies, most were 
single-generation feeding trials in which no adverse effects were noted, but in three rat studies where saccharin was fed in utero followed by lifetime feeding to the pups, an increase in bladder tumours was noted in the males. These findings were reported as statistically significant in the studies by Munro et al. (1975) and D. L. Arnold (unpublished results) but not in the study conducted by the Wisconsin Alumni Research Foundation Institute in 1972. Although no other long-term adverse findings were detected there was a tendency to proteinurea and microcalculi in the young rats and these findings may support the hypothesis that physical irritation was a contributing factor in the production of bladder tumours in the two-generation studies.

Contrary to most other food additives there have been many epidemiological trials conducted attempting to relate saccharin consumption with bladder cancer. Howe et al. (1977) and Miller \& Howe (I977) recently reported increases in bladder cancer in males but a concomitant decrease in females, thus giving rise to a questionable association. One large-scale epidemiological survey being conducted in the USA is now complete but still undergoing analysis of results. $3000-4000$ individuals with newly diagnosed bladder cancer in 1978 have been examined together with 6000 control cases. Initial analyses of the results have indicated that the findings are consistent with the associations seen in the experiments in laboratory animals but more analyses have yet to be performed.

Thus after many years of use the safety of saccharin is in question. Its carcinogenic potential has been neither proven nor disproven. Added to this the results of mutagenicity tests are equivocal. The potential withdrawal of saccharin in 1977 caused such consternation in the USA that public opinion was sufficient for the FDA to suspend its plans for banning the sweetener and to merely, at this stage, place hazard warnings on foods containing saccharin. In this way the onus seemingly is on the consumer to decide whether or not he wishes to take the risk of consuming a potentially hazardous food additive.

\section{Nitrates, nitrites and nitrosamines}

The problems caused by the association of nitrites and nitrosamines with cancer are far more complex than for many other food additives because their precursors, nitrate and nitrite, respectively, are common constituents of the normal diet. The nitrate ion is essential for plant growth and occurs in soil either naturally or added in the form of fertilizer. Soil-derived nitrate may be consumed by man in the form of plant material, and especially in those concentrating nitrate such as celery, spinach and beet, and from drinking water. Apart from the possibility of thyroid effects, nitrate is generally accepted as being non-toxic. In man nitrate is absorbed from the small intestine, it is secreted in the saliva and gastric juice and excreted in the urine. It is readily reduced to nitrite by bacterial action both during storage of nitrate-containing food, especially when the food is not refrigerated, and within the human body. Up to IO-I $2 \mathrm{mg}$ nitrite/d may be produced by the body from saliva alone. It is nitrite from all these sources, together with that deliberately added to foods in its capacity as a preservative, that presents a toxic hazard to man due to 
(I) direct toxicity of nitrite (methaemoglobinaemia in children), (2) formation of Nnitroso compounds (nitrosamines and nitrosamides) following reaction between nitrite and secondary tertiary amines and amides.

Nitrite is known to cause methaemoglobinaemia but its carcinogenic potential is questionable. A study was completed in 1978 in which levels of up to 2000 parts/million nitrite were given to rats in various food and water media (Newberne, 1979). A non-dose-related but statistically significant increase in 'lymphomas' was detected in the treated rats (10.2\% incidence compared with $5.4 \%$ in the controls). This is the first study in which nitrite itself has been connected with carcinogenicity. Unfortunately, the study is open to serious criticism and is currently being reassessed by UAREP (Universities Associated for Research and Education in Pathology).

On the other hand the carcinogenic effects of nitrosamines have been very well documented in recent years. However, many of the studies have incorporated levels of administration far in excess of the calculated likely human exposure to these materials. Recently, though, studies with comparable levels of administration have indicated that male rats and mice appear to be more susceptible to the carcinogenic effects of a variety of nitrosamines. The results of one study are still undergoing statistical evaluation and it is hoped to carry out a time-related analysis to estimate the risk attached from the likely human exposure of $\mathrm{I} \mu \mathrm{g}$ nitrosamines $/ \mathrm{kg}$ per $\mathrm{d}$.

Several workers have tried to correlate the incidence of stomach cancer with nitrate and nitrite and subsequent nitrosamine exposure. The highest rates of stomach cancer occur in Japan and Chile. Two correlating aspects of these findings are that Japanese people eat a very high concentration of dried and salted foods many of which may concentrate nitrite, whilst the world's largest nitrate mines are in Chile. However, establishing a positive causal relationship will take many more years of effort.

Bearing the above in mind the question must be considered particularly by legislative bodies; 'where do all these studies leave us?' Nitrates occur naturally in the environment thus even the banning of nitrite as a food additive will not prevent the ultimate formation of nitrosamines from nitrate reduced in the body to nitrite and reacting with secondary or tertiary amines derived from natural proteins consumed in the diet; nor will it prevent the questionably carcinogenic effects of nitrite itself. In the meantime the important point to consider is the extent to which nitrate and nitrite used as food preservatives add significantly to the normal body burden of nitrite. If this is shown to be minimal, the benefits obtained in preventing the incidence of botulism must far outweigh their 'risks' as food additives.

\section{Carcinogenicity testing}

The methods used to test food additives for potential carcinogenicity have evolved much in recent years. Long-term tests were designed initially to assess whether there were any effects on the appearance, behaviour, survival, growth, clinical biochemistry (blood, serum and urine) and gross and histopathology. Of all 
the parameters monitored less attention was paid to pathology and often not all the animals were examined; but as pathological techniques and methods for statistical analyses improved, greater numbers of animals were examined and the chances of detecting a carcinogenic effect increased. The last decade saw the design of specific carcinogenicity studies. These included the use of large groups of animals some of which were killed at intermediate intervals during the study in order to determine any effects on tumour latency. Nowadays long-term tests in animals are normally designed to study both the chronic toxic effects of a compound together with its potential carcinogenic effects and as such are large-scale and extremely expensive studies. Recently, much criticism has been levelled at the quantities of test materials given to the experimental animals compared with the known or likely human intake (as with saccharin for example). Substances should be administered at realistic levels with one low dose level approximating to the likely human intake and other dose levels as multiples of this. The use of 'maximum tolerated doses' may produce results which are difficult to interpret. It should be noted that the feeding of excessively high levels of a compound may result in a different metabolic pathway being followed compared with that followed after low-level administration. This latter point is important in cases where man and the test species share the same route of metabolism.

There are many problems involved in the design and interpretation of results from carcinogenicity studies, particularly those relating to food additives; (I) the possibility that young animals may have an increased susceptibility to carcinogens has led to some study designs including exposure in utero followed by lifetime feeding of the pups, thus giving transplacental exposure for compounds with the ability to cross the placental barrier. The need for this type of approach has not yet been established although it would mimic the human situation if the levels of administration in the animal studies were comparable with those in man, (2) the interpretation of results obtained from inbred strains of animals poses many questions. The main problem arising from the use of such animals is the possibility of selection of a line which may be particularly resistant to the test material. Therefore more than one line should be used in the animal experiments. There is also much debate on whether strains with a high or low 'spontaneous' tumour incidence should be used, (3) the question of the extent to which short-term in vitro screening tests should be used in predicting potential carcinogenicity is very important at the present time. Currently the use of a range of screening tests serves as a useful guide in this prediction but as yet there is no group of in vitro tests available which could reliably replace the conventional long-term testing in animal species. Vast quantities of time and resources are being devoted to this particular area and every encouragement should be given to the development of suitable screens.

\section{Conclusions}

There have been few instances where the carcinogenic potential of a food additive has been clearly determined in animal experiments and on these occasions 
the additive has been immediately withdrawn. If not it would contravene the Food and Drugs Act, 1955, which states that a food should not be injurious to health. There have been no documented cases of food additives causing cancer in man. Needless to say there have probably been countless potential food additives which, when tested for carcinogenicity, would have exhibited a consumer risk that the manufacturers decided against marketing.

This paper has highlighted several points which may be summarized as follows: (I) It is important to maintain good laboratory practice at all times and especially to ensure that pathological studies are first class. There is much need at present for training in this field. (2) Many food additives are naturally occurring substances and if a positive association is made between the substance and cancer in experimental animals, consumption of the material cannot necessarily be prevented altogether, although it could be reduced. This also bears out the point that 'natural' is not necessarily 'safe'. (3) The compound under test should always be given by the route to which man is or will be exposed for a valid extrapolation of results from experimental animals to be made. With the case of food additives, oral administration is required. (4) It is important to establish at an early stage in the testing of a compound the routes of metabolism in the test animals and man (if possible). This would ensure the appropriate tests being carried out in the most suitable laboratory animal. (5) Use can be made of in vitro screening tests in predicting possible carcinogenicity; these results, if negative, should not be relied upon solely but should be supported by the results of well-conducted long-term carcinogenicity studies. (6) It is important that statistical analyses are correctly selected and applied to the results available. (7) In most food applications more than one food additive is available that could fulfil a required role. But in cases where this is not so, and a possible hazard to human health is indicated, the evalution must weigh up the risks of its consumption versus the benefits it gives the human population by virtue of its use and at the same time, industry should aim to develop a safer, suitable alternative. (8) The evaluation of food additives, and for that matter, all other substances, would be facilitated further by good lines of communication and active collaboration between government and industry. (9) Where possible the design for carcinogenicity tests should be standardized to enable comparison of results from all studies performed.

The safety-in-use of food additives is closely monitored in the UK and other countries worldwide, and with the present laws the consumer is well protected against any deleterious effects of food additives. With the improvements in testing methodology the consumer can only benefit to an even greater extent in the future.

\section{REFERENCES}

Benedetti, M. S., Malnoë, A. \& Broillet, A. L. (I977). Toxicology 7, 69. Borchert, P., Miller, J. A., Miller, E. C. \& Shires, T. K. (1973). Cancer Res. 33, 590. Cohen, S. M. (1977). Gann 68, 473.

Grasso, P., Gangolli, S. D., Golberg, L. \& Hooson, J. (1971). Fd Cosmet. Toxicol. 9, 463. Grasso, P. \& Golberg, L. (1966). Fd Cosmet. Toxicol. 4, 297.

Homburger, F., Kelley, T., Friedler, G. \& Russfield, A. B. (I961). Med. Exp. 4, I. 
Howe, G. R., Burch, J. D., Miller, A. B., Morrison, B., Gordon, P., Weldon, L., Chambers, L. W., Fodor, G. \& Winsor, G. M. (1977). Lancet ii, 578.

Imahori, A., Yanagida, Y., Yamamoto, K., Shiobara, S. \& Kitagawa, T. (1977). F. Fd Hyg. Soc. fap. 18,455 .

Kinebuchi, M., Kawachi, T., Matsukura, N. \& Sugimura, T. (1979). Fd Cosmet. Toxicol. 17, 339.

Long, E. L., Nelson, A. A., Fitzhugh, O. G. \& Hansen, W. H. (1963). Archs Path. 75, 595. Miller, A. B. \& Howe, G. R. (1977). Lancet ii, 1221.

Munro, I. C., Moodie, C. A., Krewski, D. \& Grice, H. C. (1975). Toxic. appl. Pharmac. 32, 513.

Newberne, P. M. (1979). Science N.Y. 204, 1079.

Sano, T., Kawachi, T., Matsukura, N., Sasajima, K. \& Sugimura, T. (1977). Z. Krebsforsch. 89, $6 \mathrm{r}$.

Takayama, S. \& Kuwabara, N. (1977). Cancer Letters 3, II5.

Wisconsin Alumni Research Foundation Institute (1972). Preliminary report-Chronic toxicity study-sodium saccharin, presented in New York 1972.

Wislocki, P. G., Miller, E. C., Miller, J. A., McCoy, E. C. \& Rosenkranz, H. S. (1977). Cancer Res. 37, 1883 .

Yokoro, K., Kajihara, H., Kodama, Y., Nagao, K., Hamada, K. \& Kinomura, A. (1977). Gann 68, 825 . 